\title{
Wireless Channel Modeling for Leadless Cardiac Pacemaker: Effects of Ventricular Blood Volume
}

\author{
Pritam Bose, Ali Khaleghi, Senior Member, IEEE, Ilangko Balasingham, Senior Member, IEEE
}

\begin{abstract}
In this paper, the effects of ventricular blood volume change on the cardiac wireless channel modeling at 2.4 $\mathrm{GHz}$ are presented for future multi-node leadless capsule pacemakers. Numerical electromagnetic simulations are implemented in the anatomical model of human body and experiments are done in a homogeneous liquid phantom. The results show that the coupling between the capsule antennas decreases with increasing blood volume, as the blood is a highly attenuating medium. The received power varies by $3.1 \mathrm{~dB}$ for the implant-to-implant link inside the heart and varies by 1.3 $\mathrm{dB}$ for the heart implant to sub-cutaneous implant, during the diastole and systole cycle. The findings are useful to develop an intra-body communication between leadless pacemakers providing timing information for synchronized pacing in a multi-chamber leadless pacemaker system.
\end{abstract}

\section{INTRODUCTION}

Leadless cardiac pacemaker is an innovative technology that can be a replacement to the widely used pacemaker technology with leads [1]. This can help to eliminate the can-related infections and lead-related complications [2-4]. There are currently two commercially available leadless pacing systems: the Nanostim leadless cardiac pacemaker (LCP) device (St. Jude Medical, Sylmar, California) [5] and the Micra Transcatheter pacing system (TPS) (Medtronic, Minneapolis, Minnesota) [6]. Both these technologies offer single-chamber stimulation but the technology providing multi-chamber stimulations and cardiac resynchronization will be an optimum solution [7]. Recent research introduces the multi-nodal leadless pacemaker technology where each unit will hereinafter be called capsules (see Fig. 1). All the capsules will be equipped with radio-frequency (RF) communication module to develop a sensor network. To build the RF communication network, the in-body channel models inside the heart and surrounding the heart need to be clearly understood. To the best of our knowledge, very little research has been done to characterize the in-body channel models $[8,9]$. Moreover, the channel models vary depending on the position of the implant inside the body and with the frequency of operation. The channel models also vary with

This work was funded by the European Union's H2020: MSCA: ITN program for the "Wireless In-body Environment Communication WiBEC" project under the grant agreement no. 675353 .

Pritam Bose is with the Intervention Center, Oslo University Hospital, NO-0027 Oslo and also with the Faculty of Medicine, University of Oslo, 0315, Oslo, Norway (e-mail: pritam.bose@ @studmed.uio.no).

Ali Khaleghi and Ilangko Balasingham are with the Intervention Center, Oslo University Hospital, NO-0027 Oslo and with the Department of Electronic Systems, Norwegian University of Science and Technology, $7491 \quad$ Trondheim, Norway (e-mail: ali.khaleghi@rrresearch.no and ilangko.balasingham@ @edisin.uio.no). the change in blood volume inside the chambers of the heart during the cardiac cycle.

The body is a heterogeneous medium consisting of frequency dependent lossy tissues having different permittivity and conductivity. Tissues such as blood and muscle having high water content causes more attenuation of RF signals compared to fat or bone with low water content. Our research focuses on the effects of ventricular blood volume change during the cardiac cycle on the wireless channel model for the cardiac implant to implant communication, and cardiac implant to sub-cutaneous implant communication using numerical simulations and phantom experiments. The channel models are obtained at Industrial, Scientific and Medical (ISM) band at $2.4 \mathrm{GHz}$. The frequency selection is done because of easy implementation of a very small antenna providing reasonable radiation efficiency and matching, as reported by us in [10]. In addition, the possibility of decreasing the physical size of the antenna and the embedded electronics provides us more space for a larger sized battery, thereby increasing the lifetime of the pacemaker device.

The paper is organized as follows: Section II, describes the methods used in the study, fabrication of the components and the experimental setup to study RF propagation from the implants. Description of our experimental results is provided in Section III. Finally, concluding remarks and future directions are expressed in Section IV.

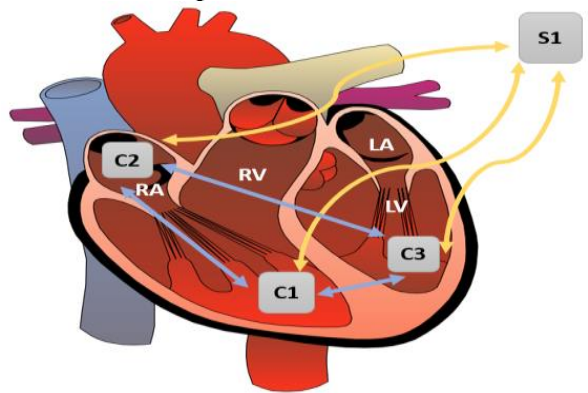

Fig. 1 Schematic of a three-node leadless capsule pacemaker application for cardiac resynchronization therapy. The capsules $\mathrm{C} 1, \mathrm{C} 2$ and $\mathrm{C} 3$ are placed inside the chambers of the heart and they can communicate with the subcutaneous implant S1. RA- right atrium, RV- right ventricle, LA- left atrium, LV- left ventricle, C- capsules and S- subcutaneous implant.

\section{Methodology AND FABRICATION}

During the heartbeats, the volume of the blood inside the heart chambers constantly vary, between end-diastole when the heart is dilated with maximum blood volume and endsystole when the heart is contracted and contains minimum volume. The blood is a highly dispersive medium due to the high mineral content. The blood volume in the right 
ventricle varies approximately between $14 \mathrm{~mL}$ to $180 \mathrm{~mL}$ for an adult heart between end-systole and end-diastole [11]. To simulate how the variation of blood volume affects the attenuation of signal, we designed an electromagnetic simulation scenario.

The electromagnetic simulations are performed using a 3D electromagnetic simulation tool of CST- Microwave Studio (https://www.cst.com/) [12]. The voxel data sets mimicking the dielectric properties of human body tissues are used for the calculations of Maxwell equations in the complex medium. The anatomical dataset used in our calculations is obtained from the Visible Human Project [13, 14] and the voxel model developed from it in CST is known as HUGO model. The model is based on a dissected male corpse sliced into several thousand layers, which are sampled and interpolated together, creating a highly detailed computational model. The import is performed via an interface that allows different resolutions $(1 \mathrm{~mm}-8 \mathrm{~mm})$ and different material tissues to be selected. The resolution of $1 \mathrm{~mm}$ by $1 \mathrm{~mm}$ is used for the simulations in this study. The high resolution is the requirement for accurate computations, as the wavelength at $2.4 \mathrm{GHz}$ in the biological medium is short and we should apply small voxel sizes.

The model is very detailed and considers the dielectric properties of each biological material separately. The dielectric properties of individual types of human body tissues are modeled to mimic the changes in the permittivity and conductivity values with respect to frequency. The upper torso of the HUGO model starting from the lower base of the neck to the upper abdomen is used for simulation. The fullbody simulation is not done in the HUGO model since it is computationally expensive and not required for the estimation of the channel models inside- and close to the heart.

A blood-containing sphere is placed inside the right ventricle of the heart of the HUGO model and the diameter of the sphere is varied in proportion to the ventricular blood flow (see Fig. 2). The simulation is run for sphere radiuses of 15, 20, 25 and $35 \mathrm{~mm}$ which accounts for 14, 33, 65 and $180 \mathrm{~mL}$ of blood respectively. Capsule $\mathrm{C} 1$ is placed in the right ventricle, $\mathrm{C} 2$ in the left ventricle and $\mathrm{S} 1$ in the shoulder subcutaneously. The capsules are modeled as simple dipole antennas with a length of $5 \mathrm{~mm}$ and a diameter of $2 \mathrm{~mm}$ enclosed on all sides in a vacuum tube of width $1 \mathrm{~mm}$. The vacuum gap of $1 \mathrm{~mm}$ is provided on all sides to prevent the direct contact of the antenna with the body tissues. The length of $5 \mathrm{~mm}$ is a rational size for the antenna, whereas the rest of the space of the capsule will be occupied by other components. For the capsule to be implanted transvenously using current catheters, the length of the capsule is limited to $10 \mathrm{~mm}$ for the multi-node pacemaker technology. The size of required antennas and battery restricts available space for sensors and actuators to be incorporated in such device, which often necessitates a trade-off between bandwidth for transmission, data rates, power requirements and the size of the device. The simulations are performed to investigate the change in the received signal power at $2.4 \mathrm{GHz}$ during the

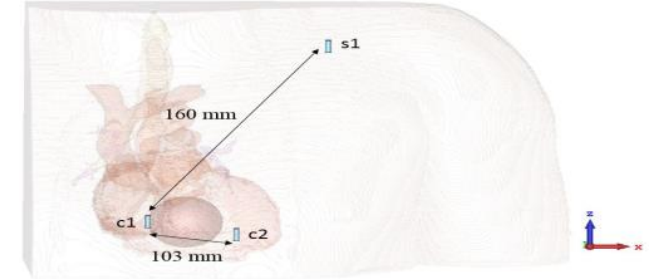

Fig 2. Capsules are placed in the right ventricle (C1), left ventricle (C2) and shoulder (S1). A sphere containing blood is placed in the right ventricle whose volume is changed to simulate the different ventricular blood volumes during the cardiac cycle

cardiac cycle.

The dipole antennas are always oriented along the z-axis which is in the coronal plane of the human body. The normalization of the S-parameters is done to remove the mismatch effects of the transmitter antenna (Tx) and receiver antenna ( $\mathrm{Rx})$ and to find the coupling between the antennas [15]. The normalization of the power received by $\mathrm{Rx}$ from Tx is calculated using,

$$
\left|S_{21}\right|_{\text {norm }}=\frac{\left|S_{21}\right|}{\sqrt{1-\left|S_{11}\right|^{2}} \sqrt{1-\left|S_{22}\right|^{2}}}
$$

where $\left|S_{21}\right|_{\text {norm }}$ is the normalized power received, $S_{21}$ is the power received before normalization, $S_{11} \& S_{22}$ are the return losses of the Tx and Rx, respectively.

The Tx mismatch is removed using,

$$
\left|\mathrm{S}_{11}\right|_{\text {norm }}=\frac{\left|\mathrm{S}_{11}\right|}{1-\left|\mathrm{S}_{11}\right|^{2}}
$$

where $\left|\mathrm{S}_{11}\right|_{\text {norm }}$ is the normalized return loss of the Tx and $\left|S_{11}\right|$ is the return loss before normalization.

The results from the mathematical simulation of the cardiac implant to sub-cutaneous implant communication are validated by an in-vitro experiment in a phantom solution. Our phantom model is a $39.2 \%$ homogeneous sucrose solution that mimics the permittivity and conductivity of human heart at $2.4 \mathrm{GHz}$ [16]. The liquid phantom is stored in a plastic container of size $24 \times 30 \times 30 \mathrm{~cm}^{3}$.

The transmitter capsule (Tx) is placed inside the liquid phantom by attaching to a plastic rod. The transmitter capsule consists of mainly three components: printed circuit board (PCB), antenna and battery (see Fig. 3a). The capsule is enclosed in an air-tight plastic container to prevent any leakage of external fluids inside the capsule during the experiments which can damage the antenna and the electronics. Moreover, it prevents the leakage of conducting charges from the antenna and also reduces the sensitivity of the entire distribution of current to the electrical properties of the ambient medium. The antenna is $1 \mathrm{~mm}$ radius meander-shaped with a resonant frequency of $2.4 \mathrm{GHz}$ which is matched at that frequency. The PCB has a voltagecontrolled oscillator (VCO) IC (HMC385LP4E) operating at $3 \mathrm{~V}$ and generating a sinusoidal signal at $2.4 \mathrm{GHz}$. The output power is $+4.5 \mathrm{dBm}$. The received power is normalized to obtain the coupling between $\mathrm{Tx}$ and $\mathrm{Rx}$ 


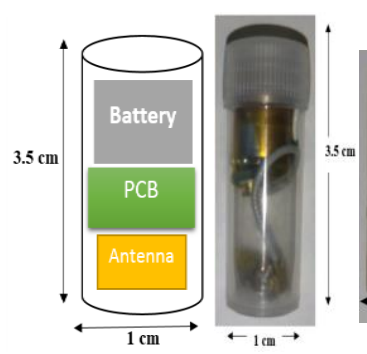

(a) (b)

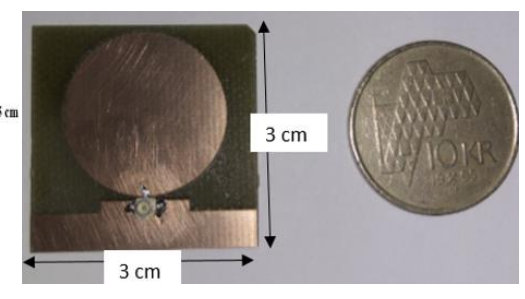

Fig. 3. (a) The schematic of the capsule and the photograph of the transmitter implant capsule containing the $\mathrm{PCB}$, battery and meander antenna b) sub-cutaneous implant patch antenna

antennas. The battery has a capacity of $160 \mathrm{mAh}$ supplying a constant voltage of $3 \mathrm{~V}$ to the $\mathrm{VCO}$.

The receiver capsule $(\mathrm{Rx})$ contains a wide-band patch antenna of dimensions $3 \mathrm{~cm} \times 3 \mathrm{~cm}$ having a return loss of $11 \mathrm{~dB}$ at $2.4 \mathrm{GHz}$ (see Fig. 3b). The antenna is encapsulated using plastic covering to prevent direct contact with tissues. It is connected to the spectrum analyzer to record the received signal power.

$\mathrm{Tx}$ and $\mathrm{Rx}$ capsules are placed inside the phantom solution at the distance of $9 \mathrm{~cm}$ (see Fig. 4a). The received power at Rx is recorded using a spectrum analyzer. A thin rubber balloon is filled with fresh liquid blood of a pig. The balloon takes the shape of a sphere having a diameter of 4 $\mathrm{cm}$. The balloon is then placed in the center between the Tx and Rx capsules (see Fig. 4b). The Rx received power is recorded in the presence of the sphere containing blood. The process is repeated by pouring more blood in the balloon to have the diameter of the sphere as $5 \mathrm{~cm}$ and $6 \mathrm{~cm}$ respectively. Multiple readings are taken at all the steps to obtain an average of the received power to minimize the chances of human errors. The received power is then normalized to obtain the coupling between $\mathrm{Tx}$ and $\mathrm{Rx}$ antennas.

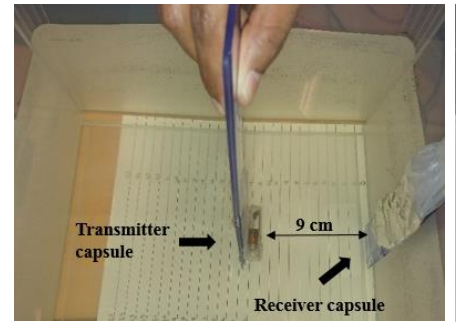

(a)

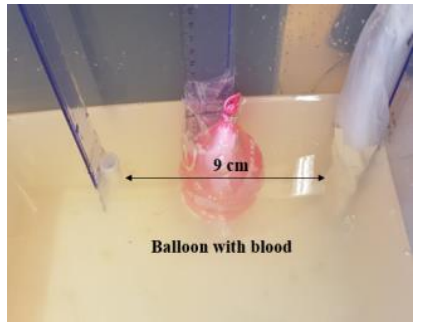

(b)
Fig. 4 Heart phantom solution with a) transmitter and receiver capsules placed inside it b) sphere containing blood placed between the transmitter and receiver capsule.

\section{MEASUREMENT RESUlTS AND DisCUSSIONS}

The coupling between the capsule antennas changes with the change in blood volume. The simulation results show that a coupling of $-81.8 \mathrm{~dB},-82.7 \mathrm{~dB},-83.1 \mathrm{~dB}$ and $-84.9 \mathrm{~dB}$ between the $\mathrm{C} 1$ and $\mathrm{C} 2$ capsules at $2.4 \mathrm{GHz}$ when the sphere contains $14 \mathrm{~mL}, 33 \mathrm{~mL}, 65 \mathrm{~mL}$ and $180 \mathrm{~mL}$ of blood respectively (see Fig. 5a). So, the coupling varies by $3.1 \mathrm{~dB}$ between the diastole and systole of heart. The coupling between $\mathrm{C} 1$ and $\mathrm{S} 1$ capsules at $2.4 \mathrm{GHz}$ are $-127.6 \mathrm{~dB}$, $127.9 \mathrm{~dB},-128.7 \mathrm{~dB}$ and $-128.9 \mathrm{~dB}$ when the sphere contains $14 \mathrm{~mL}, 33 \mathrm{~mL}, 65 \mathrm{~mL}$ and $180 \mathrm{~mL}$ of blood respectively (see Fig. 5b). So, the coupling between the heart implant and the subcutaneous implant varies by $1.3 \mathrm{~dB}$ between the diastole and systole of heart. The variation of coupling with the change in ventricular blood volume is more significant in communication between heart implants than heart to subcutaneous implant. This is because the sub-cutaneous implant is located at a greater distance from the heart implant and most of the signal passes through tissues which have less attenuation than blood. Moreover, the blood volume is varied in only one chamber of the heart whereas in real scenarios, the blood volume would vary in all the chambers of the heart combined with cardiac movements.

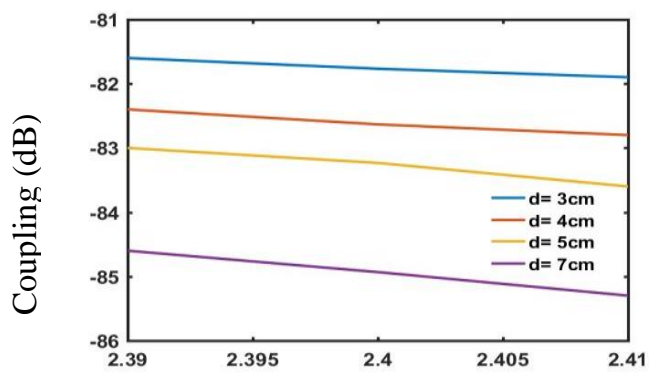

(a)

Frequency $(\mathrm{GHz})$

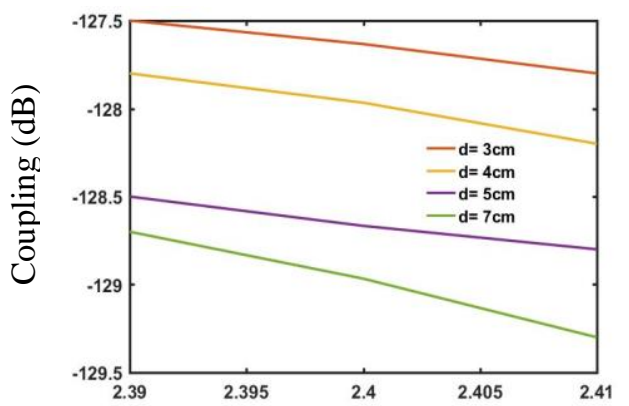

(b)

Frequency $(\mathrm{GHz})$

Fig. 5 Coupling in $\mathrm{dB}$ between the transmitter and receiver antennas in frequency range of $2.39 \mathrm{GHz}$ to $2.41 \mathrm{GHz}$ in the HUGO model. a) The transmitter capsule $\mathrm{C} 1$ is placed in the right ventricle close to the apex and receiver capsule $\mathrm{C} 2$ is placed in the left ventricle b) The transmitter capsule $\mathrm{C} 1$ is placed in the right ventricle close to the apex and receiver capsule S1 is placed in the shoulder subcutaneously

The phantom experimental setup is prepared to compare the simulation results with the phantom results. The coupling between $\mathrm{Tx}$ and $\mathrm{Rx}$ of $-71.5 \mathrm{~dB},-76 \mathrm{~dB}$ and $-79.5 \mathrm{~dB}$ is recorded at $2.4 \mathrm{GHz}$ when the balloon contains $31 \mathrm{~mL}, 67$ $\mathrm{mL}$ and $121 \mathrm{~mL}$ of blood respectively (see Table I). The coupling decreases by $4 \mathrm{~dB}, 6 \mathrm{~dB}$ and $10 \mathrm{~dB}$ with the introduction of $31 \mathrm{~mL}, 67 \mathrm{~mL}$ and $121 \mathrm{~mL}$ of blood respectively. Thus, the phantom results confirm the simulation results that the coupling decreases with the increases in blood volume as blood is a highly attenuating medium. The most efficient communication period will be during the end-systole due to lower attenuation of signal in that time-period. The signal attenuation difference of $10 \mathrm{~dB}$ between the end-systole and end-diastole is quite significant in case of in-body communication system. 
TABLE I COUPLING IN DB BETWEEN THE TRANSMITTER AND RECEIVER ANTENNAS AT 2.4 GHZ PLACED IN THE PHANTOM SOLUTION WITHOUT THE PRESENCE OF BLOOD AND BY VARYING THE BLOOD VOLUME INSIDE THE BALLOON

\begin{tabular}{|c|c|c|c|c|}
\hline $\begin{array}{c}\text { Distance } \\
\text { between } \\
\text { antenna }\end{array}$ & $\begin{array}{c}\text { Diameter of } \\
\text { balloon }(\mathrm{cm}) \\
/ \text { Blood } \\
\text { volume } \\
(\mathrm{mL})\end{array}$ & $\begin{array}{c}\text { Coupling } \\
\text { without } \\
\text { blood } \\
(\mathrm{dB})\end{array}$ & $\begin{array}{c}\text { Coupling } \\
\text { with blood } \\
(\mathrm{dB})\end{array}$ & $\begin{array}{c}\text { Difference } \\
(\mathrm{dB})\end{array}$ \\
\hline $9 \mathrm{~cm}$ & $4 / 31$ & -72 & -76 & 4 \\
\hline $9 \mathrm{~cm}$ & $5 / 67$ & -74.5 & -80.5 & 6 \\
\hline $9 \mathrm{~cm}$ & $6 / 121$ & -74 & -84 & 10 \\
\hline
\end{tabular}

The objective of the study is not exactly to correlate the simulation results with the phantom experiment results but to validate the hypothesis that the coupling between the implants decreases with increase in ventricular blood volume. Both the numerical simulations and phantom experiments proves it which is quite encouraging. This will help us to find out the appropriate timing of the transmission of signals between the implants. The results cannot be directly correlated due to different contributing factors. The simulation considers an ideal environment whereas the experimental setup involves interference from surrounding environment. An ideal dipole antenna is used for the numerical simulations whereas in case of the phantom experiments, a meander-shaped antenna of different length is used for the heart implant and a patch antenna for the receiver implant to collect the received signal. The sphere containing blood is used for the simulation whereas the balloon containing blood is not exactly spherical. The balloon has a bulge towards the bottom due to the weight of the blood inside. The phantom experiment is done by attaching the antennas to plastic rods and placing it in the phantom solution, so the results are slightly influenced by small human errors. The numerical simulation is performed in a heterogeneous human voxel model with biological tissues having different dielectric properties whereas the phantom solution is a homogeneous one having the average dielectric properties of the human heart. The other contributing factors are impedance mismatch of the antennas, antenna rotation, polarization mismatch and misalignment of the antenna in the phantom model.

\section{CONCLUSION AND FUTURE WORK}

The effects of ventricular blood volume change on the inbody channel model are presented in this paper for the development of multi-chamber leadless pacemaker technology. The phantom experiments are in good agreement with the computer simulation results supporting the hypothesis that the coupling between the implants decreases with increase in ventricular blood volume. We have also stated that the most efficient communication period will be during the end-systole due to lower attenuation of signal in that time-period. The future work would involve performing experiments on living animals. The simulation results provide a rational basis for animal experiments, help better planning and possibly reduce the number of such experiments. The animal experiment would provide more realistic results as the signals will be subjected to time-varying shadowing, reflection, and diffraction effects due to the heart movements during the cardiac cycle. The simulations and the phantom experiments are static scenarios where the antennas are fixed at a distance whereas the dynamic scenario in the living animal experiment would cause movements of the capsule resulting in different distances between them at different stages of the cardiac cycle. Thus, further experiments would facilitate in the design of a complete prototype of the multinode leadless capsule pacemaker technology.

\section{ACKNOWLEDGMENT}

This work was funded by the European Union's H2020: MSCA: ITN program for the "Wireless In-body Environment Communication - WiBEC" project under the grant agreement no. 675353 .

\section{REFERENCES}

[1]M. Sweeney, A. Hellkamp, and K. Ellenbogen, "Adverse effect of ventricular pacing on heart failure and atrial fibrillation among patients with normal baseline QRS duration in a clinical trial of pacemaker therapy for sinus node dysfunction," ACC Current Journal Review, vol. 12, no. 5, pp. 65-66, Jun 2003.

[2] M. S. Kiviniemi, M. A. Pirnes, H. J. K. Eranen, R. V. Kettunen, and J. E. Hartikainen, "Complications Related to Permanent Pacemaker Therapy," Pacing \& Clinical Electrophysi., vol. 22, no. 5, pp. 711-720, May 1999.

[3] A. A. Harcombe, S. A. Newell, P. F. Ludman, T. E. Wistow, L. D. Sharples, P. M. Schofield, D. L. Stone, L. M. Shapiro, T. Cole, and M. C. Petch, "Late complications following permanent pacemaker implantation or elective unit replacement," Heart, vol. 80, no. 3, pp. 240-244, Jan. 1998.

[4] K. G. Tarakji, O. M. Wazni, S. Harb, A. Hsu, W. Saliba, and B. L. Wilkoff, "Risk factors for 1-year mortality among patients with cardiac implantable electronic device infection undergoing transvenous lead extraction: the impact of the infection type and the presence of vegetation on survival," Europace, vol. 16, no. 10, pp. 1490-1495, Feb. 2014.

[5]C.-P. Lau, K. Chen, K.-F. Lee, Y. Dai, and S. Zhang, "Implantation and Clinical Performance of an Entirely Leadless Cardiac Pacemaker," International Journal of Heart Rhythm, vol. 1, no. 1, p. 50, Sep. 2016.

[6]E. H. Nichols and P. Ritter, "Micra Transcatheter Pacing System Safe, Effective," MD Conference Express, vol. 15, no. 22, pp. 8-9, Jan. 2015.

[7]S. Sideris, S. Archontakis, P. Dilaveris, K. A. Gatzoulis, K. Trachanas, I. Sotiropoulos, P. Arsenos, D. Tousoulis, and I. Kallikazaros, "Leadless Cardiac Pacemakers: Current status of a modern approach in pacing," Hellenic Journal of Cardiology, vol. 58, no. 6, pp. 403-410, May 2017

[8] K. Takizawa, T. Aoyagi, J.-I. Takada, N. Katayama, K. Yekeh, Y. Takehiko, and K. R. Kohno, "Channel models for wireless body area networks," 2008 30th EMBC, pp. 1549-1552,Aug. 2008.

[9] Li, Z. Nie, Y. Liu, L. Wang and Y. Hao, "Characterization of In-Body Radio Channels for Wireless Implants", IEEE Sensors Journal, vol. 17, no. 5, pp. 1528-1537, Mar. 2017.

[10]P. Bose, A. Khaleghi, M. Albatat, J. Bergsland and I. Balasingham, "RF Channel Modeling for Implant to Implant Communication and Implant to Sub-Cutaneous Implant Communication for Future Leadless Cardiac Pacemakers", IEEE Transc. on Biomedical Engg., Mar. 2018 (in press).

[11] A. M. Maceira, S. K. Prasad, M. Khan, and D. J. Pennell, "Reference right ventricular systolic and diastolic function normalized to age, gender and body surface area from steady-state free precession cardiovascular magnetic resonance," European Heart Journal, vol. 27, no. 23, pp. 2879-2888, Nov. 2006.

[12]Microwave Studio, "CST-Computer Simulation Technology." Bad Nuheimer Str, vol. 19,pp. 64289, 2008.

[13]M. Ackerman, "The Visible Human Project," Proceedings of the IEEE vol. 86, no. 3, pp. 504-511, Mar. 1998.

[14]V. Spitzer, M. Ackerman, A. Scherzinger and D. Whitlock, "The Visible Human Male: A Technical Report", Journal of the American Medical Informatics Association, vol. 3, no. 2, pp. 118-130, Mar. 1996.

[15]A. Khaleghi and I. Balasingham, "Power coupling for conceptual antennas in medical implant applications," 11th European Conference on Antennas and Propagation (EUCAP), pp. 1864-1867, Mar. 2017.

[16]S. Castello-Palacios, A. Valles-Lluch, C. Garcia-Pardo, A. Fornes-Leal, and N. Cardona, "Formulas for easy-to-prepare tailored phantoms at 2.4 GHz ISM band,” 2017 (ISMICT), pp. 27-31, Feb. 2017. 